\title{
Macular Edema Formation and Deterioration of Retinal Function after Intravitreal Bevacizumab Injection for Proliferative Diabetic Retinopathy
}

\author{
Hisanori Imai ${ }^{\mathrm{a}, \mathrm{b}}$ Shigeru Honda ${ }^{\mathrm{a}}$ Yasutomo Tsukahara ${ }^{\mathrm{a}}$ \\ Akira Negi ${ }^{a}$ \\ aDivision of Ophthalmology, Department of Organ Therapeutics, Kobe University \\ Graduate School of Medicine, and 'b Department of Ophthalmology, Kobe Kaisei \\ Hospital, Kobe, Japan
}

\section{Key Words}

Anti-vascular endothelial growth factor - Bevacizumab · Avastin - Multifocal electroretinography · Full-field electroretinography - Diabetic retinopathy - Optical coherence tomography $\cdot$ Macular edema

\begin{abstract}
Purpose: To report a case of proliferative diabetic retinopathy (PDR) showing transient macular edema (ME) and deteriorated retinal function after intravitreal bevacizumab injection (IVB).
\end{abstract}

Methods and Results: A 53-year-old man received IVB $(1.25 \mathrm{mg} / 0.05 \mathrm{ml})$ in both eyes for the treatment of PDR. There was no treatment-related complication. However, he complained of photopsia in both eyes $6 \mathrm{~h}$ after the injection. Slit-lamp examination revealed mild cellular infiltrations (1+) in the anterior chamber in both eyes. Optical coherence tomography showed ME formation in the left eye. Both full-field and multifocal electroretinography (ERG) revealed the deterioration of all parameters in both eyes compared with pretreatment. The inflammation in the anterior segment and ME disappeared 1 day after the injection. ERG parameters were improved 9 days after the injection, except for the $\mathrm{N} 1$ and $\mathrm{P} 1$ amplitude of multifocal ERG in the left eye.

Conclusion: We propose that patients who undergo IVB should be carefully informed and followed up for possible complications including temporal ME formation and retinal function deterioration. 


\section{Introduction}

A number of reports described the benefit of intravitreal bevacizumab (Avastin ${ }^{\circledR}$; Genentech, Inc., South San Francisco, Calif., USA) injection (IVB) for proliferative diabetic retinopathy (PDR) [1-3]. Excessive fibrosis of fibrovascular membrane (FVM) and tractional retinal detachment after IVB are reported to date [4-6]. In this report, we present a case of PDR showing transient macular edema (ME) and deteriorated retinal function after IVB.

\section{Case Report}

A 53-year-old man consulted our hospital for the treatment of PDR in both eyes. At the first visit, the best corrected visual acuity measured by Landolt ring chart was 1.0 in both eyes. No remarkable finding was observed in the anterior segment. Fundoscopic examination revealed normal macular appearance and apparent FVM on the disc and elsewhere. Scattered chorioretinal scars from retinal photocoagulation performed more than 3 months before were also found. Fluorescein angiography showed remarkable leakages of dye from the FVM (fig. 1a). However, the optical coherence tomography (OCT) findings showed normal macular appearances (fig. 2). To reduce the activity of FVM, we performed off-label IVB $(1.25 \mathrm{mg} / 0.05 \mathrm{ml})$ in both eyes with the patient's written informed consent, the approval of the institutional review board of Kobe University of Medicine and the tenets of the Declaration of Helsinki. Although no complication occurred during the surgical procedures, the patient complained of photopsia in both eyes $6 \mathrm{~h}$ after the injection. Slit-lamp examination revealed mild cellular infiltrations (1+) in the anterior chamber in both eyes. Although the fundoscopic findings in both eyes did not reveal any retinal change, the OCT showed ME formation in the left eye (fig. 2). Both full-field and multifocal electroretinography (ERG) revealed the deterioration of all parameters in both eyes compared with pretreatment (fig. 3). The next day, the inflammation in the anterior segment and ME disappeared (fig. 2), and after 9 days, the patient's complaint had totally disappeared. Best corrected visual acuity was maintained at 1.0 in both eyes during the event. Fluorescein angiography revealed leakage reduction (fig. 1b). Moreover, 9 days after the injection, ERG parameters were improved, except for the N1 and P1 amplitude of multifocal ERG in the left eye (fig. 3).

\section{Discussion}

Although Fung et al. [7] published the incidence of ocular inflammation related to IVB, they did not evaluate the structural change of the retina after the injection. To our knowledge, this is the first report describing ocular inflammation accompanied by ME shortly after IVB. It is known that ME usually results from accelerated breakdown of the blood-retinal barrier due to PDR. However, in our case, apparent ME occurred quite rapidly after IVB, which suggests that ME was induced by the surgical procedure or by bevacizumab itself. Forooghian et al. [8] reported aqueous vascular endothelial growth factor (VEGF) downregulation and the upregulation of inflammatory cytokines (TGF- $\beta 2$ and IL-8) after IVB. They discussed that acute elevation of these inflammatory cytokines may mediate a rapid contraction of FVM, thereby causing tractional retinal detachment. Since there is no report demonstrating such a rapid appearance of ME after IVB, we suggest that the acute alteration of the intraocular cytokine concentration might have had a predominant role in inducing inflammatory ME in the present case.

The ERG findings showed a functional deterioration after IVB. Changes of all ERG parameters were determined after only $6 \mathrm{~h}$ following IVB, corresponding to the slit-lamp and OCT findings, but the recovery of the ERG parameters was delayed compared to the improvement seen in the slit-lamp and OCT findings. This result indicates that 
subclinical dysfunction of the retina lasts relatively longer than morphological changes. Peters et al. [9] showed the reduction of choriocapillaris endothelial cell fenestrations after IVB, and Heiduschka et al. [10] showed the penetration of bevacizumab through the retina and diminished immunoreactivity of VEGF in the retina 14 days after IVB. They suggested that these ultrastructural changes may lead to functional and morphologic damage of retinal pigment epitheliums and photoreceptors. We considered that the VEGF withdrawal after IVB suppressed the physiological neurotrophic effect of VEGF [11], and caused temporal retinal dysfunction accompanied by the abnormal ERG parameters.

In summary, we propose that patients who undergo IVB should be carefully informed and followed up for possible complications including temporal ME formation and retinal function deterioration.
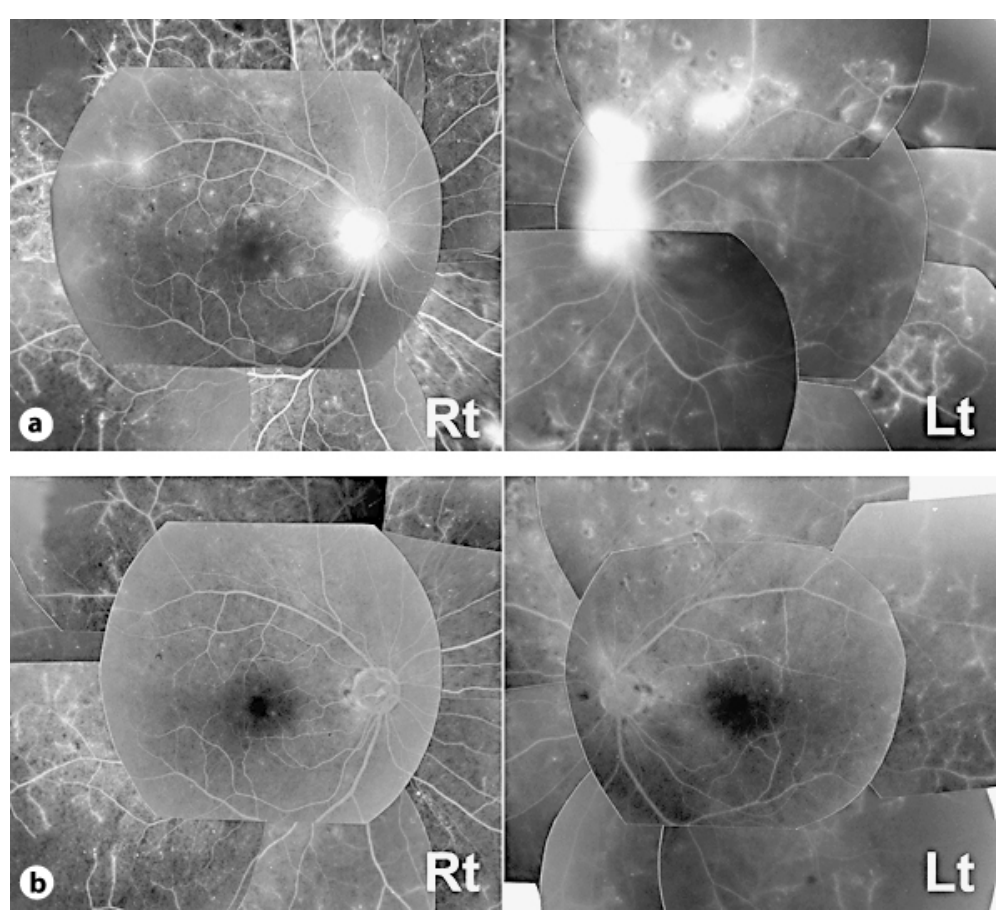

Fig. 1. Late-phase fluorescein angiography findings. a Leakages from neovascularizations on the disc and elsewhere were seen before IVB in both eyes. $\mathbf{b}$ Leakages from neovascularizations were reduced 9 days after the injection. 

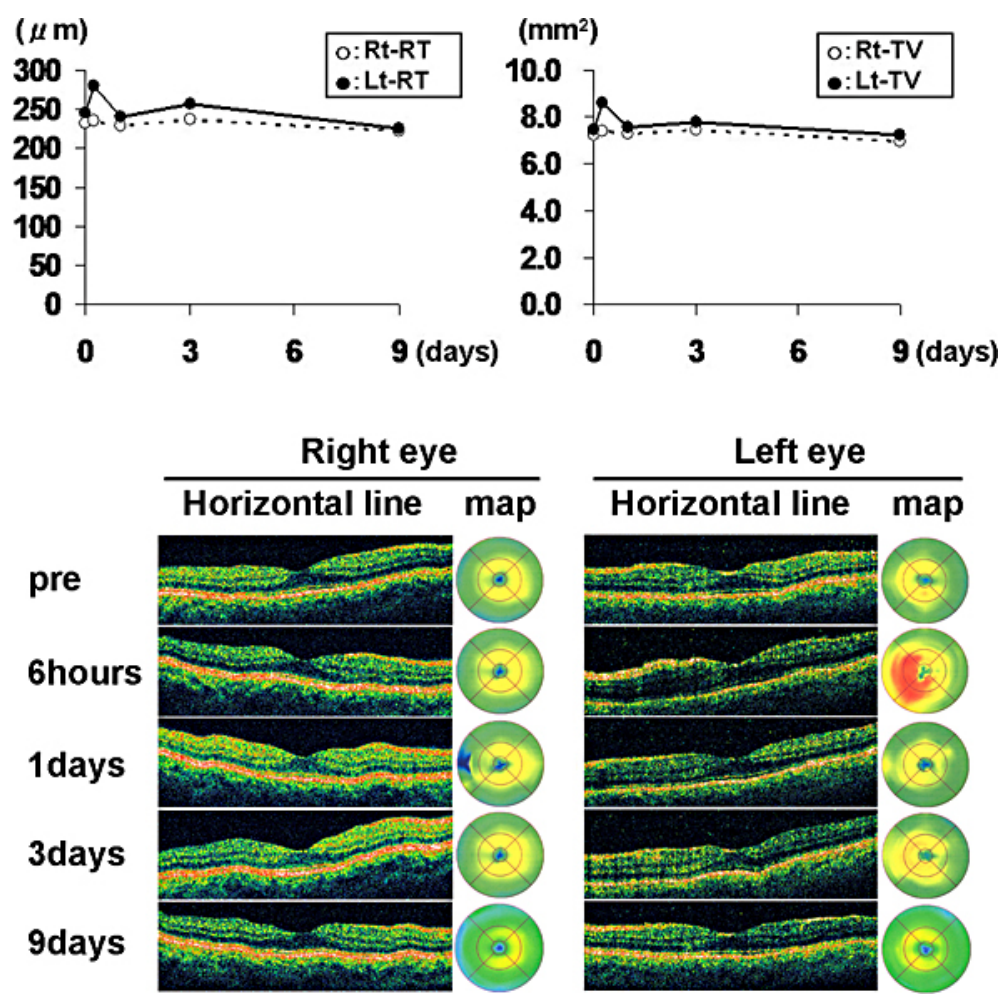

Fig. 2. Upper panel: transition of retinal thickness and total macular volume in OCT. Lower panel: transition of the horizontal line and the color map image of retinal thickness in OCT. Before the injection, OCT findings showed normal macular appearances in both eyes. The retinal thickness and total macular volume in the left eye were increased $6 \mathrm{~h}$ after the injection. OCT findings in the left eye improved after 1 day and were kept normal after that. OCT findings in the right eye were kept normal during the follow-up period. 

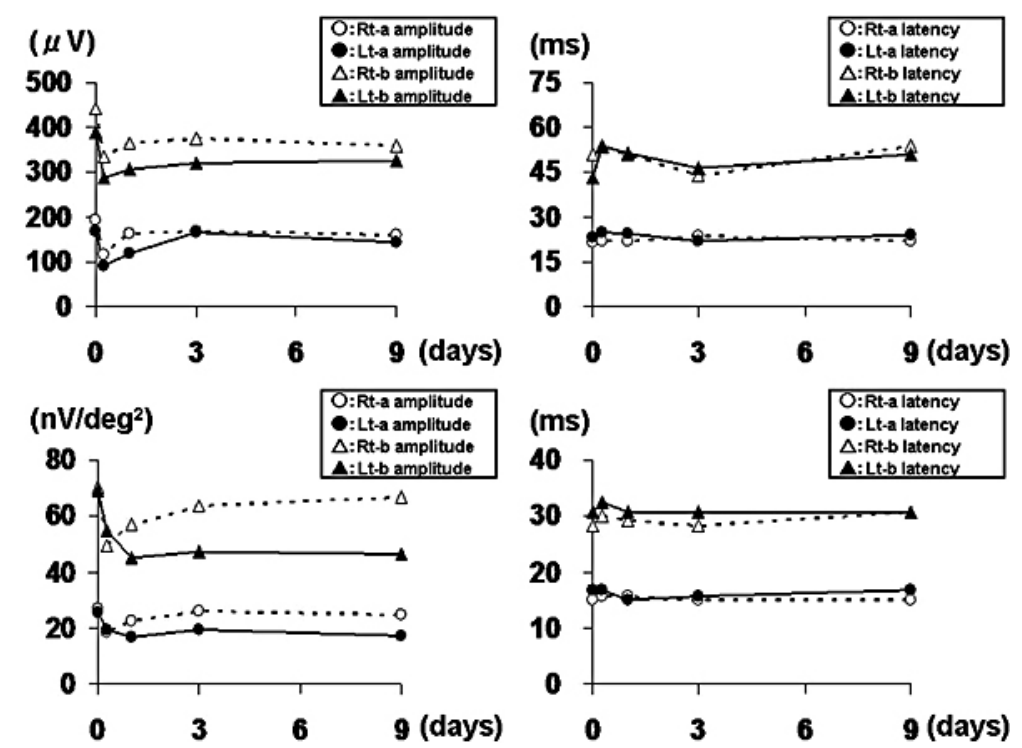

Fig. 3. Transition of ERG parameters. Upper panel: transition of full-field bright-flash ERG. Lower panel: transition of multifocal ERG at 3 central rings of 103 hexagon analysis. In both methods, all ERG parameters worsened $6 \mathrm{~h}$ after the injection and improved with time, except for the N1 and P1 amplitudes of the left eye.

\section{References}

1 Arevalo JF, Wu L, Sanchez JG, et al: Intravitreal bevacizumab (avastin) for proliferative diabetic retinopathy: 6months follow-up. Eye 2009;23:117-123.

-2 Ishikawa K, Honda S, Tsukahara Y, Negi A: Preferable use of intravitreal bevacizumab as a pretreatment of vitrectomy for severe proliferative diabetic retinopathy. Eye 2009;23:108-111.

-3 Modarres M, Nazari H, Falavarjani KG, Naseripour M, Hashemi M, Parvaresh MM: Intravitreal injection of bevacizumab before vitrectomy for proliferative diabetic retinopathy. Eur J Ophthalmol 2009;19:848-852.

-4 Arevalo JF, Maia M, Flynn HW Jr, Saravia M, Avery RL, Wu L, Eid Farah M, Pieramici DJ, Berrocal MH, Sanchez JG: Tractional retinal detachment following intravitreal bevacizumab (Avastin) in patients with severe proliferative diabetic retinopathy. Br J Ophthalmol 2008;92:213-216.

-5 Jonas JB, Schmidbauer M, Rensch F: Progression of tractional retinal detachment following intravitreal bevacizumab. Acta Ophthalmol 2009;87:571-572.

-6 Tranos P, Gemenetzi M, Papandroudis A, Chrisafis C, Papadakos D: Progression of diabetic tractional retinal detachment following single injection of intravitreal Avastin. Eye 2008;22:862.

7 Fung AE, Rosenfeld PJ, Reichel E: The International Intravitreal Bevacizumab Safety Survey: using the internet to assess drug safety worldwide. Br J Ophthalmol 2006;90:1344-1349.

8 Forooghian F, Kertes PJ, Eng KT, Agrón E, Chew EY: Alterations in the intraocular cytokine milieu after intravitreal bevacizumab. Invest Ophthalmol Vis Sci 2010;51:2388-2392.

9 Peters S, Heiduschka P, Julien S, et al: Ultrastructural findings in the primate eye after intravitreal injection of bevacizumab. Am J Ophthalmol 2007;143:995-1002.

10 Heiduschka P, Fietz H, Hofmeister S, et al: Penetration of bevacizumab through the retina after intravitreal injection in the monkey. Invest Ophthalmol Vis Sci 2007;48:2814-2823.

11 Brockington A, Lewis C, Wharton S, Shaw PJ: Vascular endothelial growth factor and the nervous system. Neuropathol Appl Neurobiol 2004;30:427-446. 